\title{
Synergetic Contributions in Phase Boundary Engineering to the Piezoelectricity of Potassium Sodium Niobate Lead-Free Piezoceramics
}

Xiang Lv ${ }^{1,2}$, Junwei Zhang ${ }^{2}$, Yao Liu ${ }^{3}$, Fei Li ${ }^{3}$, Xi-xiang Zhang ${ }^{2 *}$, and Jiagang $\mathrm{Wu}^{1 *}$

${ }^{1}$ Department of Materials Science, Sichuan University, Chengdu 610065, P. R. China

${ }^{2}$ Division of Physical Science and Engineering, King Abdullah University of Science and Technology (KAUST), Thuwal 239955, Kingdom of Saudi Arabia

${ }^{3}$ Electronic Materials Research Laboratory, Key Laboratory of Education Ministry/International Center for Dielectric Research, Xi'an Jiaotong University, Xi'an 710049, P. R. China

*Correspondence e-mail: wujiagang0208@163.com and msewujg@scu.edu.cn(J.W); xixiang.zhang@kaust.edu.sa (X-x.Z)

\begin{abstract}
Although the pronounced piezoelectricity was obtained in $(\mathrm{K}, \mathrm{Na}) \mathrm{NbO}_{3}$ piezoceramics with the phase boundary engineering (PBE), the physical mechanisms remain pending. Here we revealed for the first time how PBE influences the piezoelectric properties through synergetic contributions. Cryogenic experiments confirm that PBE constructs a phase coexistence, consisting of rhombohedral $(\mathrm{R})$, orthorhombic $(\mathrm{O})$, and tetragonal $(\mathrm{T})$ phases, with a structural softening, by which a high piezoelectric coefficient $d_{33}$ of $555 \mathrm{pC} / \mathrm{N}$ and the enhanced temperature stability of strain are achieved. The phenomenological theory and transmission electron microscope demonstrate that the superior $d_{33}$ hinges on the flattened Gibbs free energy and the abundant nano-domains (10-80 nm), which respectively induce the enhanced permittivity and the coexisting single-domain and multi-domain zones. In particular, we disclosed a trade-off relationship between ferroelectric domains and polar
\end{abstract}


nanoregions (PNRs) and found the "double-edged sword" role of PNRs in the piezoelectricity enhancement. Therefore, this work helps understand the physical mechanisms of the piezoelectricity enhancement, benefiting the future research of lead-free piezoceramics.

Keywords: Potassium sodium niobate; Phase boundary engineering; Structural softening; Nanodomains; Physical mechanisms

\section{Introduction}

Demand for replacing the toxic lead-based piezoceramics has made investigations on lead-free piezoceramics one of the hottest topics in ferro/piezoelectric. ${ }^{1}$ Potassium sodium niobate $\{(\mathrm{K}$, $\mathrm{Na}) \mathrm{NbO}_{3}, \mathrm{KNN}$ - -based ceramics attract researchers' attention because of the high Curie temperature $\left(T_{\mathrm{c}} \sim 410{ }^{\circ} \mathrm{C}\right)$ and moderate piezoelectricity. ${ }^{2}$ In particular, the newly proposed phase boundary engineering (PBE), simultaneously adjusting two ferroelectric-ferroelectric phase transition temperatures (i.e., rhombohedral-orthorhombic $T_{\mathrm{R}-\mathrm{O}}$ and orthorhombic-tetragonal $T_{\mathrm{O}-\mathrm{T}}$ ) to room temperature, effectively enhanced the piezoelectric properties and improved the temperature stability, ${ }^{3-}$ ${ }^{6}$ endowing KNN-based ceramics with the promising potential in practical application. ${ }^{7}$

Although the PBE brought in the pronounced piezoelectricity, the physical mechanisms remain pending, specifically insufficiently understanding the synergetic contributions from the phase structure, ferroelectric domains, and polar nanoregions (PNRs). ${ }^{8}$ The different phase structures were reported in the similar (or even same) KNN-based ceramics modified by the PBE. ${ }^{9-10}$ The same group even reported different phase structures when proceeding with highly similar compositions. ${ }^{5}$, 11-14 
Ferroelectric domains, the external contribution to the piezoelectricity, were reported to contribute to the piezoelectricity enhancement of KNN-based ceramics. ${ }^{4-6,15}$ However, most reported ferroelectric domains came from the unpoled samples, which have no net piezoelectricity because of lacking the poling process that causes the domain switching and domain wall motion. ${ }^{4-6,15}$ In particular, through observing domains of unpoled and poled samples, new physical mechanisms were dug in BT- and BNT-based ceramics. ${ }^{16-17}$ However, the related study is rare in KNN-based ceramics but is crucial to further analyze the physical mechanisms. ${ }^{16,18-22}$

PNRs were reported to cause distinct dielectric relaxor characteristics and contribute to the enhanced piezoelectric properties. $^{23-24}$ The PBE inevitably induces PNRs in KNN-based ceramics because of aliovalent substitutions on $\mathrm{A}-(\mathrm{K} / \mathrm{Na})$ and $\mathrm{B}-(\mathrm{Nb})$ sites,${ }^{25}$ which was revealed by the frequency dependency of permittivity. ${ }^{6,26}$ In particular, the relaxor behavior of KNN-based ceramics with PBE differs from that of canonical lead-based relaxors. ${ }^{27}$ The former occurs near room temperature together with ferroelectric-ferroelectric phase transition, but the latter occurs near $T_{\mathrm{m}}$ (the temperature of the maximum permittivity) or in the low-temperature range, accompanying with no phase transitions. ${ }^{27}$ Therefore, the different physical mechanisms are predictable. However, the role of PNRs in piezoelectricity was rarely analyzed in $\mathrm{KNN}$-based ceramics. More importantly, the piezoelectric properties of piezoceramics hinge on the synergetic effects of forgoing factors, which have never been reported in KNN-based ceramics.

Here we exampled $(1-x)\left(\mathrm{K}_{0.48} \mathrm{Na}_{0.52}\right)\left(\mathrm{Nb}_{0.955} \mathrm{Sb}_{0.045}\right) \mathrm{O}_{3}-x\left(\mathrm{Bi}_{0.5} \mathrm{Ag}_{0.5}\right) \mathrm{ZrO}_{3}-0.2 w t \% \mathrm{Fe}_{2} \mathrm{O}_{3}$ ceramics to decode the synergetic effects. We first obtained a large $d_{33}=555 \mathrm{pC} / \mathrm{N}$ and the improved temperature 
stability at $x=0.04$ where the multi-phase coexistence is. We then demonstrated the compositiondependent phase structure and the structural softening and observed ferroelectric domains of both unpoled and poled samples. Finally, we discussed the synergetic effects of the phase structure, domains, and PNRs on piezoelectric properties by combining experimental results with the phenomenological theory. The analysis shows that the superior $d_{33}$ hinges on the flattened Gibbs free energy profile and the abundant nano-domains $(10-80 \mathrm{~nm})$, which respectively induce the enhanced permittivity and the coexisting single-domain and multi-domain zones. In particular, we disclosed a trade-off relationship between ferroelectric domains and polar nanoregions (PNRs) and found the "double-edged sword" role of PNRs in the piezoelectricity enhancement.

\section{Methods}

The detailed process of preparing $(1-x)\left(\mathrm{K}_{0.48} \mathrm{Na}_{0.52}\right)\left(\mathrm{Nb}_{0.955} \mathrm{Sb}_{0.045}\right) \mathrm{O}_{3}-x\left(\mathrm{Bi}_{0.5} \mathrm{Ag}_{0.5}\right) \mathrm{ZrO}_{3}-0.2 w t \% \mathrm{Fe}_{2} \mathrm{O}_{3}$ (abbreviated as KNNS- $x$ BAZ-Fe, $x=0-0.08$ ) and measuring the phase structure, domain structure, and electrical properties can be found in the Electronic Supporting Information (ESI).

\section{Results and Discussion}

We first collected $d_{33}$ and $T_{\mathrm{c}}$ (or depolarization temperature, $T_{\mathrm{d}}$ ) of representative lead-based and leadfree piezoceramics (Figure 1(a)). Barium titanate $\left\{\mathrm{BaTiO}_{3}, \mathrm{BT}\right\}$ based ceramics exhibit a high $d_{33}$ $(620-700 \mathrm{pC} / \mathrm{N})$ but a low $T_{\mathrm{c}}\left(<100{ }^{\circ} \mathrm{C}\right) ;{ }^{28-29} \mathrm{KNN}-$ based ceramics with PBE show a high $d_{33}(490-650$ $\mathrm{pC} / \mathrm{N})$ and a benign $T_{\mathrm{c}}\left(150-250{ }^{\circ} \mathrm{C}\right) ;^{3-4,27,30}$ quenched bismuth ferrite $\left\{\mathrm{BiFeO}_{3}, \mathrm{BFO}\right\}$ based ceramics possess both a high $d_{33}(\sim 400 \mathrm{pC} / \mathrm{N})$ and a high $T_{\mathrm{c}}\left(\sim 450{ }^{\circ} \mathrm{C}\right) ;{ }^{31}$ and sodium bismuth titanate $\left\{\left(\mathrm{Bi}_{0.5} \mathrm{Na}_{0.5} \mathrm{TiO}_{3}, \mathrm{BNT}\right\}\right.$ based ceramics' $d_{33}$ and $T_{\mathrm{d}}$ are seriously limited below $300 \mathrm{pC} / \mathrm{N}$ and $200{ }^{\circ} \mathrm{C} .{ }^{32}$ 
Therefore, the PBE endows KNN-based ceramics with comparable $d_{33}$ and $T_{\mathrm{c}}$ to those of commercial piezoceramics (e.g., PZT-4 and PZT-5H) and with the promising potential for electronic devices. ${ }^{7}$ Here we obtained a high $d_{33}$ of $555 \mathrm{pC} / \mathrm{N}$ and $T_{\mathrm{c}}$ of $220^{\circ} \mathrm{C}$ at $x=0.04$, comparable to commercial soft PZT (e.g., PZT-4 and PZT-5H) ceramics and state-of-the-art non-textured KNN-based ceramics (Figure 1(a)). ${ }^{4}, 27,30$ Besides, the strain is also enhanced in the multi-phase coexistence region, and an electrostriction strain of $0.084 \%$ is obtained at $x=0.08$ (Figure S12, ESI), 10 times larger than that of morphotropic relaxor boundary (MRB)-modified KNN-BT ceramics. ${ }^{33}$

We then compared the temperature stability of the unipolar strain $\left(S_{\text {uni }}\right)$ (Figure 1(b)). BNT- and BTbased ceramics exhibit an apparent decrease with temperature. ${ }^{34-35}$ Similarly, KNN-based ceramics with an O-T phase coexistence also show a strongly temperature-dependent $S_{\text {uni. }}{ }^{36}$ Therefore, these ceramics occupy the left bottom area of Figure 1(b), showing the relatively poor temperature stability. The almost vertical morphotropic phase boundary (MPB) endows PZT-4 and PZT-5H ceramics with excellent temperature stability and the increased $S_{\text {uni }}$ with temperature. ${ }^{37}$ Thus, lead-based ceramics take up the top area of Figure 1(b). However, the PBE significantly enhances temperature stability and thus helps KNN-based ceramics fill up the gap between the abovementioned lead-free and lead-based ceramics. In particular, samples with $x=0.04$ show comparable temperature stability to KNN-BLT-BZMn ceramics and highly textured KNN-3T ceramics (Figure 1(b)). ${ }^{5-6}$ Therefore, the PBE indeed endows KNN-based ceramics with high $d_{33}$ values and improved temperature stability, guaranteeing the reliability of subsequent analysis and discussion. 

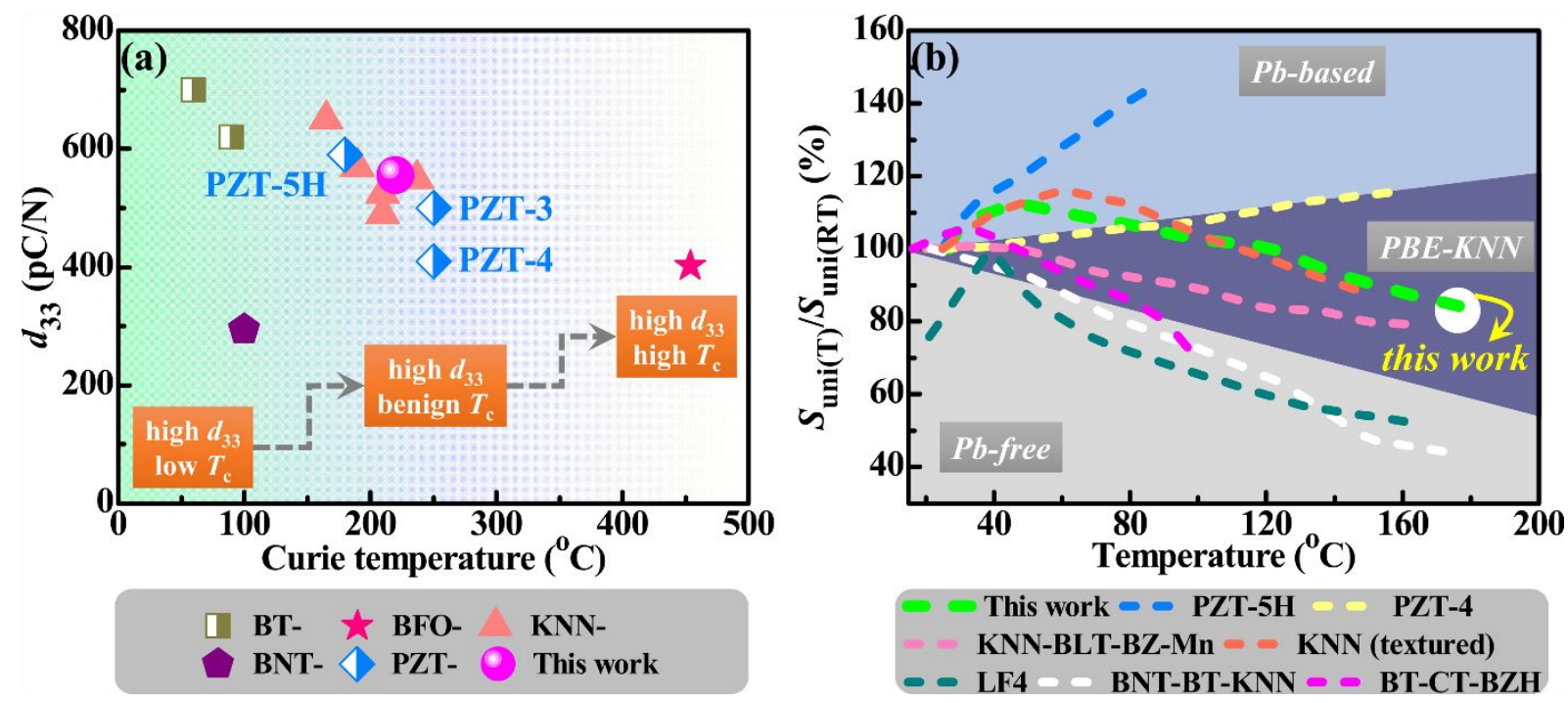

Figure 1. (a) Relationship between $d_{33}$ and $T_{\mathrm{c}}$ (or $T_{\mathrm{d}}$ for BNT-based ceramics) in state-of-the-art non-textured leadfree piezoceramics; (b) $S_{\text {uni(T) }} / S_{\text {uni(RT) }}$ varying with temperature in representative lead-based and lead-free piezoceramics. This work means the temperature stability of $S_{\text {uni }}$ of KNNS- $x$ BAZ-Fe $(x=0.04)$ ceramics (Figure S13, ESI).

To resolve the controversy for the phase structure of KNN-based ceramics with PBE, we first analyzed the composition-dependent phase structure by the combined tools (Figures S1-S9, ESI). In particular, we carried out the systemic temperature-dependent dielectric properties (i.e., real part of permittivity $\varepsilon^{\prime}-T$, imaginary part of permittivity $\varepsilon^{\prime \prime}-T$, and loss factor tan $\delta$ - $T$ ) for all compositions for the first time, which were only conducted on the particular a few compositions in most references. ${ }^{6,12,38} \mathrm{We}$ demonstrated that samples with $x=0.04$ possessed an R-O-T multi-phase coexistence whether before or after poling (Figures 2(a-c) and Figures S1-S9, ESI).

We then studied the structural softening of the R-O-T multi-phase coexistence. Temperaturedependent Raman spectra show that $v_{1}$ mode, doubly degenerated stretching mode of $\mathrm{Nb}-\mathrm{O}$ octahedron, shows two anomalies at $80{ }^{\circ} \mathrm{C}$ and $225{ }^{\circ} \mathrm{C}$, which are respectively ascribed to the R-O-T phase 
coexistence to the $\mathrm{T}$ phase and the ferroelectric to paraelectric phase transitions (Figures 2(d, e)). In particular, $v_{1}$ mode steadily decreases at $-50^{\circ} \mathrm{C}-75^{\circ} \mathrm{C}$ (Figure 2(e)), but the normal R-O and O-T phase transitions cause distinct anomalies in $v_{1}$ mode (Figures S10 (a, b), ESI). Therefore, the R-O-T multi-phase coexistence at $x=0.04$ is the diffused one, indicating the structural softening, which is absent in samples with $x=0$ because of the well-arranged lattice (Figure S10, ESI).

We next studied the origin of structural softening by measuring temperature-dependent dielectric properties at different frequencies. In unpoled samples, $\varepsilon^{\prime}-T$ and $\varepsilon^{\prime \prime}-T$ curves show the distinct relaxor characteristic at $-50^{\circ} \mathrm{C}-75^{\circ} \mathrm{C}$ (Figure 2(f, g)), and $\tan \delta$ - $T$ curves exhibit the relaxor characteristic at lower temperatures (Figure 2(h)). The relaxor characteristic here resembles that of relaxor- $\mathrm{PbTiO}_{3}$ single crystals but is accompanied by the ferroelectric-ferroelectric phase transition (or multi-phase coexistence) (Figure 2(g)). Furthermore, the poling process sharpens $T_{\mathrm{O}-\mathrm{T}}$, regains $T_{\mathrm{R}-\mathrm{O}}$ (see black arrows), and reduces the relaxor characteristic at $-50{ }^{\circ} \mathrm{C}-75^{\circ} \mathrm{C}$ (Figure 2(i-k)). The reappeared $T_{\mathrm{R}-\mathrm{O}}$ indicates that unpoled samples possess the diffused $\mathrm{R}$ phase that is then reinforced by the poling process, demonstrated by the increased $\mathrm{R}$ phase content after poling (Figure 2(c)). ${ }^{39}$ Therefore, we ascribed the structural softening to the diffused $\mathrm{R}$ phase and the relaxor characteristic associated with PNRs that are caused by aliovalent substitutions (e.g., substituting $\mathrm{Bi}^{3+}$ on $\mathrm{K}^{+} / \mathrm{Na}^{+}$and $\mathrm{Zr}^{4+}$ on $\mathrm{Nb}^{5+}$ ). Considering the low $\mathrm{R}$ phase content and its high pseudo-cubic characteristic (Table S1, ESI), we speculated that PNRs are the diffused R phase, which needs to be further studied in the future.

Even though the reduced relaxor characteristic in poled samples, $T_{\mathrm{R}-\mathrm{O}}$ is still weak, and $T_{\mathrm{O}-\mathrm{T}}$ is not as sharp as that of poled samples with $x=0$ (Figure S10, ESI). In particular, the maximum polarization 
$\left(P_{\max }\right)$ shows no abrupt decrease at $T_{\mathrm{O}-\mathrm{T}}$, and the coercive field $\left(E_{\mathrm{c}}\right)$ exhibits the slight increase at 20 ${ }^{\circ} \mathrm{C}-80{ }^{\circ} \mathrm{C}$ (Figure 2(1)), differing from samples with $x=0$ whose $P_{\max }\left(E_{\mathrm{c}}\right)$ abruptly decreases (increases) near $T_{\mathrm{O}-\mathrm{T}}$ (Figure S10, ESI). Furthermore, we found the negligible decrease in diffuseness degree $(\gamma)$ in both unpoled and poled samples by using the modified Curie-Weiss law (Figure 2(m-o) and Figure S10, ESI). Therefore, the structural softening exists at the level of the lattice of the R-O-T multi-phase coexistence and remains in poled samples. Besides, the structural softening is also proved by the temperature-dependent strain properties (Figures S13 and S14, ESI).
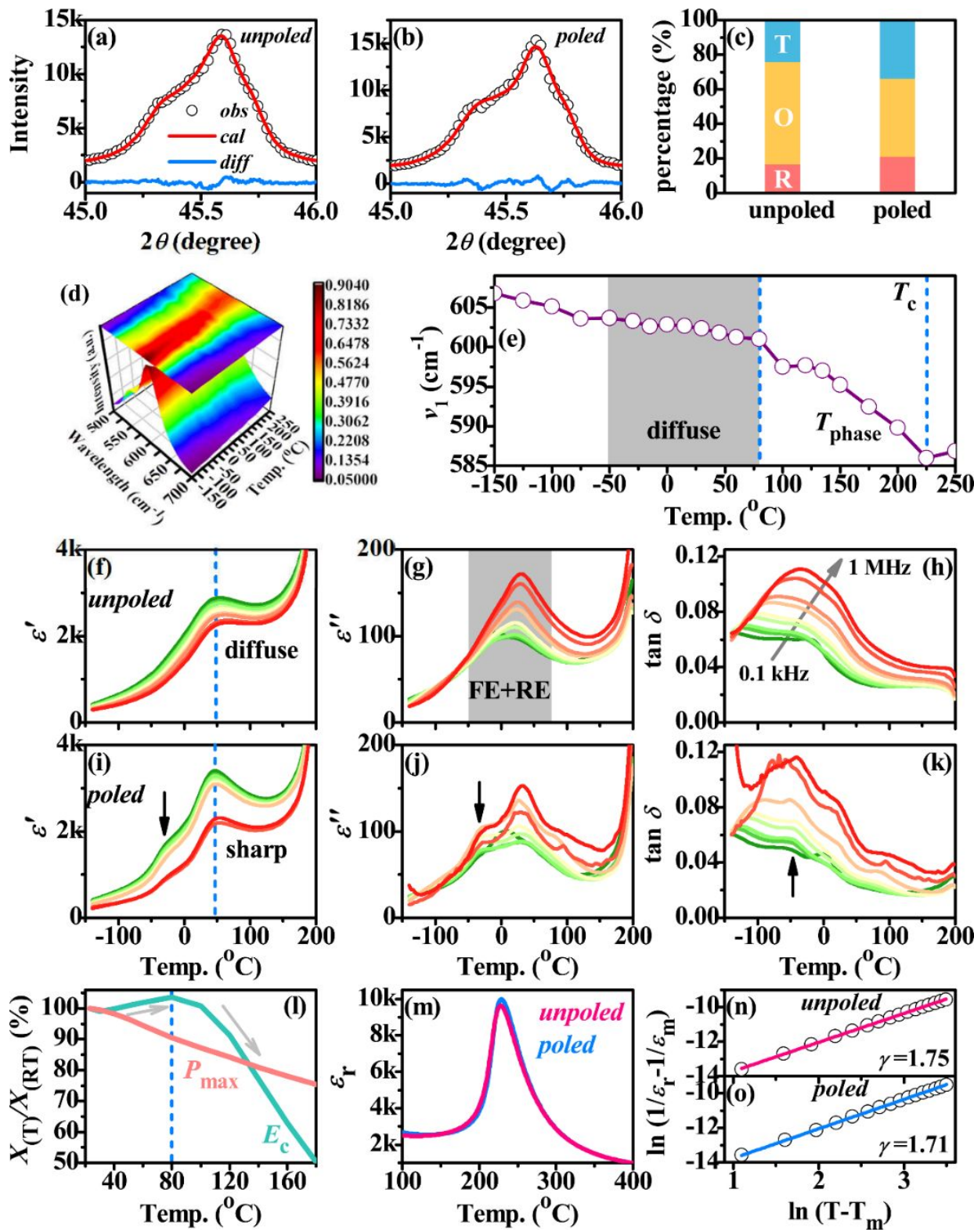

Figure 2. Phase structure and structural softening analysis for KNNS- $x$ BAZ-Fe $(x=0.04)$ ceramics. Rietveld 
refinements for (a) unpoled and (b) poled samples; (c) fraction of R, O, and T phases; (d) Temperature-dependent Raman spectra of the unpoled sample; (e) position of $v_{1}$ mode varying with temperature; (f) $\varepsilon^{\prime}-T,(\mathrm{~g}) \varepsilon^{\prime \prime}-T$, and (h) $\tan \delta$-T curves of the unpoled sample; (i) $\varepsilon^{\prime}-T$, (j) $\varepsilon^{\prime \prime}-T$, and (k) tan $\delta$-T curves of poled sample; (l) variations of $P_{\max }$ and $E_{\mathrm{c}}$ of the poled sample with temperature; (m) $\varepsilon_{\mathrm{r}}-T$ curves of unpoled and poled samples, measured at $10 \mathrm{kHz}$; linear fitting between $\ln \left(T-T_{\mathrm{m}}\right)$ and $\ln \left(1 / \varepsilon_{\mathrm{r}}-1 / \varepsilon_{\mathrm{m}}\right)$ of (n) unpoled and (o) poled samples. FE and RE respectively represent the ferroelectric multi-phase coexistence and relaxor characteristics. The black arrows in (i-k) indicate the R-O phase transition. $P_{\max }$ and $E_{\mathrm{c}}$ are extracted from Figure $\mathrm{S} 13$ (ESI).

To unveil the contribution from domains, we then conducted TEM measurements on samples with $x=0$ and $x=0.04$. Unpoled KNNS- $x$ BAZ-Fe $(x=0)$ ceramics show the herringbone submicron domains, which resemble those of other pure $\mathrm{KNN}$-based ceramics and are deemed as $90^{\circ}$ domains (Figure $\mathrm{S} 15$, ESI). ${ }^{40-41}$ Conversely, unpoled KNNS- $x$ BAZ-Fe $(x=0.04)$ ceramics exhibit much more complex domains. A rectangular-ambulatory-plane domain configuration is comprised of intensive striped nano-domains with a scale of 20-40 nm (marked as I domain in Figures 3(a, d, g)), which was also reported in other PBE-modified KNN-based ceramics with high performance. ${ }^{6,26}$ The inset of Figure 3(d) shows its structural illustration. Additionally, the striped nano-domains with a scale of 50-80 nm are also observed in the same area and are labeled as II domain (Figures 3(a, d, j)). More importantly, the intensive root-like nano-domains with a scale of 20-30 $\mathrm{nm}$ are observed (Figures $3(\mathrm{~b}, \mathrm{e}, \mathrm{h}, \mathrm{k})$ ), which heavily intertwined and formed a labyrinthine domain zone (marked as III and IV domains). ${ }^{42-}$ ${ }^{43}$ The alternative lamellar domains and curved domain walls are also observed (marked as V and VI domains) (Figures 3(c, f)), which were reported in PZT ceramics on the R phase side. ${ }^{44}$ Two sides of these alternative domains show a scale of 10-15 $\mathrm{nm}$ and 30-35 $\mathrm{nm}$ in width, respectively (Figures 3(i, 
1)). Meanwhile, each side of alternative domains is comprised of the hierarchical nano-domains and distinguished by the alternative green and red regions in Figure 3(f). Interestingly, an intermediate domain wall with a scale of $30 \mathrm{~nm}$ is also observed within some alternative domains (Figures 3(f, i)).

The inset of Figure 3(f) shows the structural illustration of these lamellar domains. The nano-domains are ascribed to the R-O-T multi-phase coexistence and are comparable to those of state-of-the-art leadfree piezoceramics..$^{5-6}$
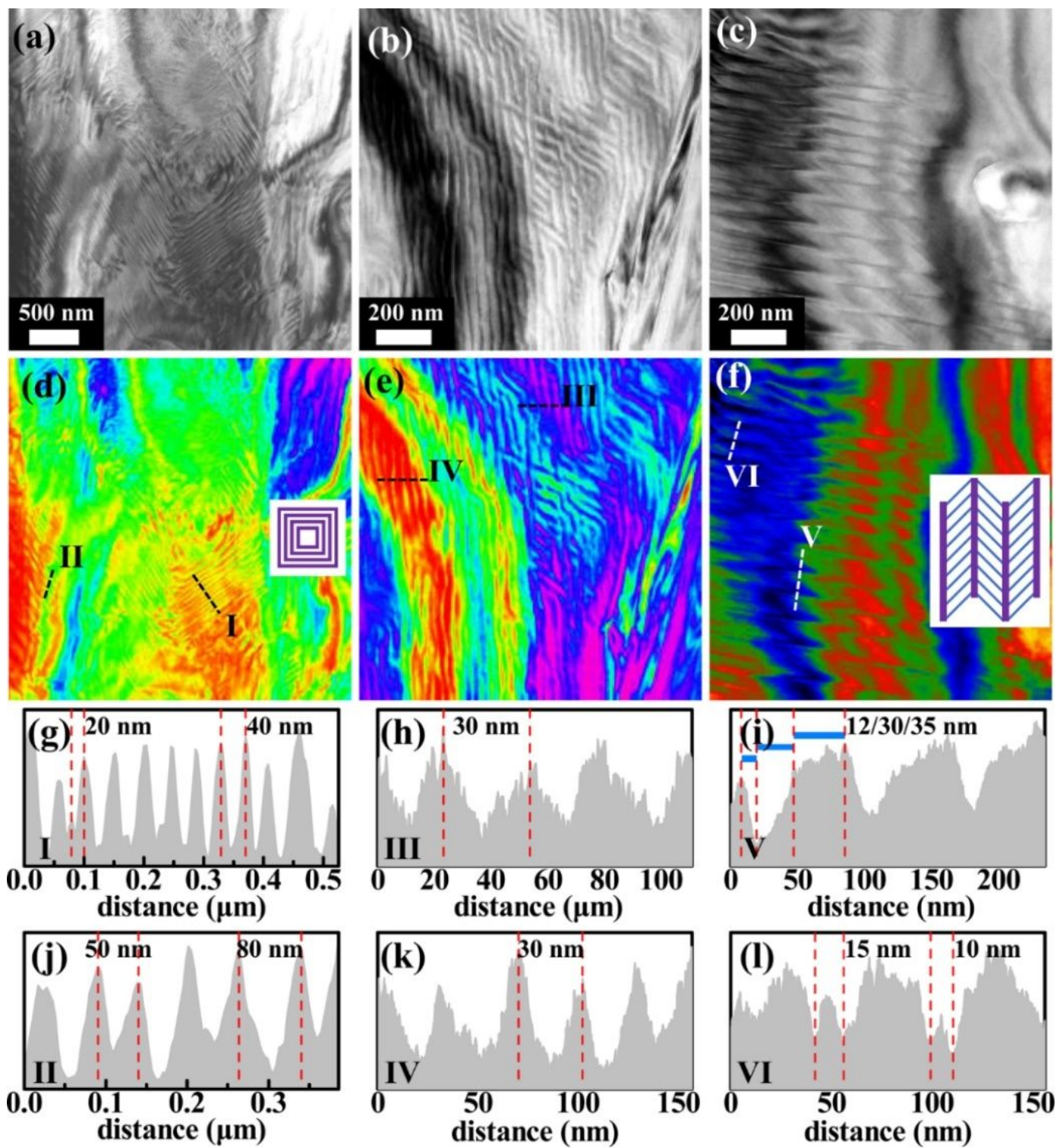

Figure 3. Domain structure of unpoled KNNS- $x$ BAZ-Fe $(x=0.04)$ ceramics. TEM image and corresponding colored image of $(a, d)$ rectangular-ambulatory-plane domain, $(b, e)$ root-like nano-domain, $(c, f)$ lamellar domain; (g-l) 
statistics of domain width of representative domains marked in (d, e, f). The insets of (d, f) show the structural illustration of rectangular-ambulatory-plane and lamellar domains, respectively.

After poling, the size and morphology of domains are greatly changed. Intensive nano-domains are found along the direction of the electric field and exhibit a scale of 20-40 nm (Figures 4(a, d, g, h)). Importantly, the coexistence of single-domain and multi-domain is observed in another grain (Figures 4(b, e)). The multi-domain configuration exhibits a scale of 80-150 $\mathrm{nm}$ (Figures 4(e, i)), and the singledomain configuration shows a flattened morphology (Figures 4(e, j)). The insets of Figures 4(b, e) show that the single-domain configurations are separated by the straight domain walls. Also, the coexistence of single-domain and multi-domain is found in other grains (Figures $4(\mathrm{c}, \mathrm{f})$ ). The multidomain configuration possesses a scale of $15-35 \mathrm{~nm}$ (Figures $4(\mathrm{f}, \mathrm{k}, 1))$. The simplification of domains is attributed to the disappearance of $108^{\circ}$ domain walls and the re-orientation of non- $180^{\circ}$ domains. ${ }^{10}$ More importantly, the unique single-domain configuration was also found in $(\mathrm{Ba}, \mathrm{Ca}) \mathrm{TiO}_{3}-\mathrm{Ba}(\mathrm{Zr}$, Ti) $\mathrm{O}_{3}$ ceramics during in situ TEM observations and believed to be responsible for the piezoelectricity enhancement. ${ }^{16,19-20}$ The initial multi-domain was changed into a single-domain after experiencing the intermediate state of nano-domains. ${ }^{16,19-20}$ The single-domain was metastable and quickly changed into the multi-domain after removing or further increasing the electric field. ${ }^{16,}$ 19-20 Therefore, the coexistence of single-domain and nanoscale multi-domain here resembles the situation of (Ba, Ca) $\mathrm{TiO}_{3}-\mathrm{Ba}(\mathrm{Zr}, \mathrm{Ti}) \mathrm{O}_{3}$ ceramics and thus contributes to the enhanced piezoelectric properties. ${ }^{16,}{ }^{19-20}$ 

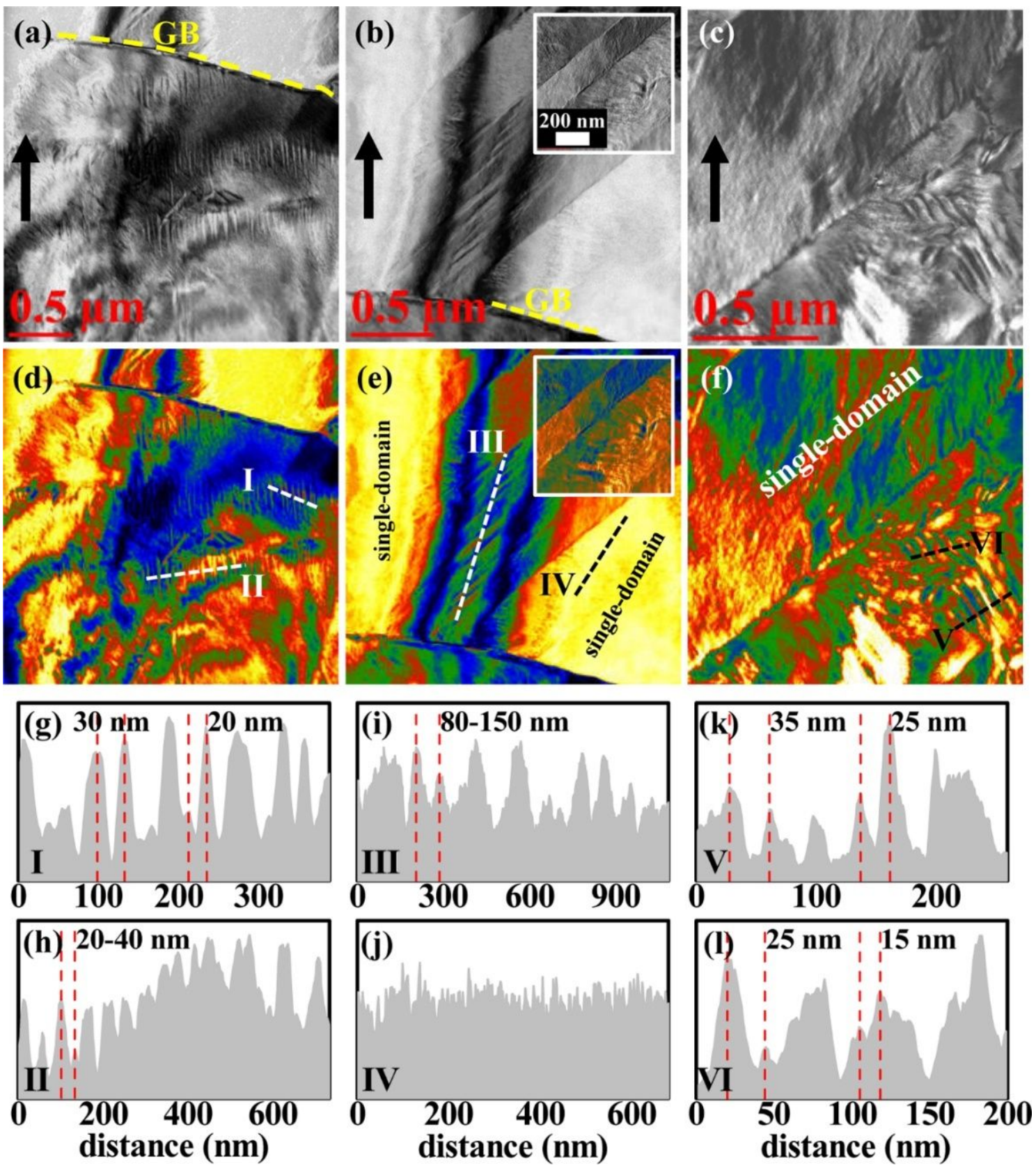

Figure 4. Domain structure of poled KNNS- $x$ BAZ-Fe $(x=0.04)$ ceramics. (a-c) TEM images and (d-f) corresponding colored images of three kinds of representative domains; (g-l) statistics of domain width of representative domains marked in ( $d, e, f)$. The black arrow in (a-c) indicates the direction of the applied electric field and "GB" in (a, b) means the grain boundary. The insets of $(b, e)$ show the enlarged view of an alternative single-domain zone.

To unveil the physical mechanisms of high $d_{33}$, we first discussed the importance of the R-O-T multiphase coexistence. Piezoelectric properties of ferroelectrics can be evaluated by the equation 


$$
d_{33}=2 Q_{33} \varepsilon_{33} P_{\mathrm{r}}
$$

where $Q_{33}, \varepsilon_{33}$, and $P_{\mathrm{r}}$ are the electrostriction coefficient, dielectric permittivity, and remanent polarization, respectively. ${ }^{45} Q_{33}$ hinges on the order degree of the cation arrangement and increases with increasing order degree. ${ }^{46}$ The PBE destroys the order degree of $\mathrm{K}^{+} / \mathrm{Na}^{+}$and $\mathrm{Nb}^{5+}$ to some degree due to aliovalent substitutions (Figures S1-S9, ESI), resulting in a reduction in $Q_{33}$. Also, $P_{\mathrm{r}}$ decreases with increasing $x$ (Figure S12, ESI). Thus, the increase in $d_{33}$ hinges on the enhanced $\varepsilon_{33}$. Indeed, both unpoled and poled samples show the increased $\varepsilon_{\mathrm{r}}$ in the R-O-T multi-phase coexistence region (Figure 5(a)). In particular, the sharp jump is observed when comparing the room-temperature $\varepsilon_{\mathrm{r}}$ in the poled samples with $x=0$ and $x=0.04$, being about the quintuple increase (Figure 5(b)). Therefore, this jump is a cornerstone for the piezoelectricity enhancement in KNNS- $x \mathrm{BAZ}-\mathrm{Fe}(x=0.04)$ ceramics. Although poled samples with $x=0.06-0.08$ still possess high $\varepsilon_{\mathrm{r}}$ because of their relatively low $T_{\mathrm{c}}$, their $P_{\mathrm{r}}$ values are significantly decreased (Figures S11-S12, ESI). Because of the synergetic effect of $\varepsilon_{\mathrm{r}}$ and $P_{\mathrm{r}}, d_{33}$ and $\varepsilon_{\mathrm{r}} P_{\mathrm{r}}$ reach their enhanced values in the R-O-T multi-phase coexistence region (Figures 5(a, d)).

The enhanced $\varepsilon_{33}$ originates from the low polarization anisotropy and the flattened Gibbs free energy (Figures 5(f-h)) $\cdot{ }^{47}$ On the one hand, the R-O-T multi-phase coexistence has more polarization vectors than the single phase and thus allows more polarization rotation paths (Figures $5(\mathrm{~d}, \mathrm{e})$ ); on the other hand, its flattened Gibbs free energy significantly reduces the low energy barrier for polarization rotation (Figures $5(\mathrm{f}-\mathrm{h})) .{ }^{47}$ Both factors promote the response of polarization to the electric field and thus enhance $\varepsilon_{33} \cdot{ }^{48-49}$ 

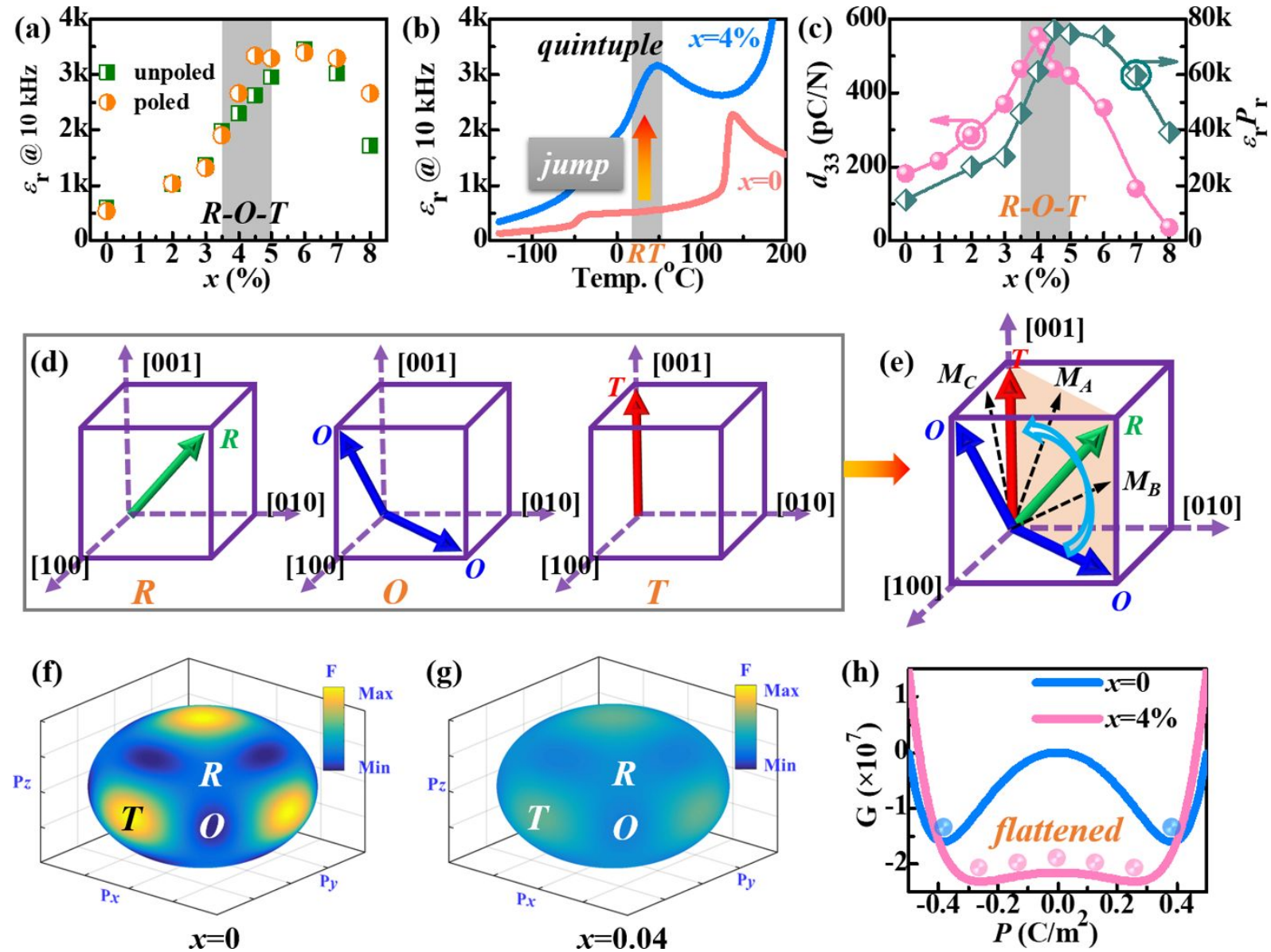

Figure 5. (a) $\varepsilon_{\mathrm{r}}$ of unpoled and poled KNNS- $x$ BAZ-Fe ceramics; (b) $\varepsilon_{\mathrm{r}}-T$ curves of poled KNNS- $x$ BAZ-Fe ceramics with $x=0$ and 0.04 ; (c) $d_{33}$ and $\varepsilon_{\mathrm{r}} P_{\mathrm{r}}$ of poled KNNS- $x \mathrm{BAZ}-\mathrm{Fe}$ ceramics; (d) structural model of spontaneous polarization of $\mathrm{R}, \mathrm{O}$, and $\mathrm{T}$ phases; (e) possible polarization rotation path in the diffused R-O-T multi-phase coexistence; 3D Gibbs free energy profile of unpoled KNNS- $x$ BAZ-Fe ceramics with (f) $x=0$ and (g) $x=0.04$; (h) twodimension (2D) Gibbs free energy profile of unpoled KNNS- $x$ BAZ-Fe ceramics ( $x=0$ and 0.04 ). For unpoled KNNS$x \mathrm{BAZ}-\mathrm{Fe}(x=0)$ ceramics, the strong polarization anisotropy is observed and the $\mathrm{O}$ phase exhibits the lowest energy, which coincides with the $\mathrm{O}$ phase structure at room temperature. For unpoled KNNS- $x \mathrm{BAZ}-\mathrm{Fe}(x=0.04)$ ceramics, the polarization anisotropy is substantially reduced and the flattened energy profile is observed among $\mathrm{R}, \mathrm{O}$, and $\mathrm{T}$ phases, indicating an easy polarization rotation at the diffused R-O-T multi-phase coexistence.

We then discussed the contributions from domains and PNRs to high $d_{33}$. According to the classical 
theory, the domain size $(d)$ is proportional to the domain wall energy $\left(F_{\mathrm{DW}}\right)$, that is, $d \propto \sqrt{F_{\mathrm{DW}}} .^{50}$ The low polarization anisotropy of the R-O-T multi-phase coexistence significantly decreases $F_{\mathrm{DW}}$ and thus reduces the domain size, resulting in the occurrence of nano-domains (Figure 3). Subsequently, these nano-domains are easily re-orientated by the electric field and then form the coexisting single-domain and multi-domain zones, promoting the piezoelectric properties (Figure 4). Furthermore, PNRs act as a seed and further promote the domain switching. ${ }^{23}$

Here we emphasized the trade-off relationship between ferroelectric domains and PNRs in the piezoelectricity enhancement. With increasing $x$, ferroelectric domains reduce in quantity and size, but PNRs show an increasing content (Figures 6(a) and Figure S11, ESI). These factors reduce the barrier energy for domain switching and domain wall motion (Figure 6(b)) and thus decreases $E_{\mathrm{c}}$ monotonously (Figures 6(c) and S12, ESI). However, the increasing (decreasing) content of PNRs (ferroelectric domains) leads to the reduction of polarization vectors along the direction of the electric field, resulting in the dramatic deterioration in $P_{\max }$ and $P_{\mathrm{r}}$ (Figures 6(c) and S12, ESI). Therefore, achieving the saturated poling becomes more difficult with increasing $x$ because of the increasing amount of non-collinear PNRs (Figure 6(a)), which is demonstrated by the decreased phase angle $(\theta)$ and the lower Raman shifting in poled KNNS- $x$ BAZ-Fe $(x=0.04)$ ceramics (Figures $6(d)$ and Figures S18-S19, ESI). Therefore, PNRs are a "double-edged sword", that is, although it facilitates the domain switching and domain wall motion, it cannot be re-orientated completely. Hence, the increased $d_{33}$ is the balanced consequence of ferroelectric domains and PNRs at the R-O-T multi-phase coexistence, which explains why $d_{33}$ and $\varepsilon_{\mathrm{r}} P_{\mathrm{r}}$ respectively reach their maximum values at $x=0.04$ and $x=0.045-0.05$ (Figure 5(c)). 
Inspired by the foregoing analysis, here we proposed a promising concept to further increase $d_{33}$ of lead-free piezoceramics. As expressed in Equation (1), both high $\varepsilon_{\mathrm{r}}$ and $P_{\mathrm{r}}$ are expected to achieve a high $d_{33}$. Therefore, an ideal concept is to introduce a small number of PNRs to a ferroelectric matrix with very strong ferroelectricity, which was realized in relaxor- $\mathrm{PbTiO}_{3}$ single crystals, ${ }^{23,51}$ but hasn't been reported in lead-free piezoelectric materials so far. Therefore, future work can focus on balancing the ferroelectric domains and PNRs in lead-free piezoceramics.
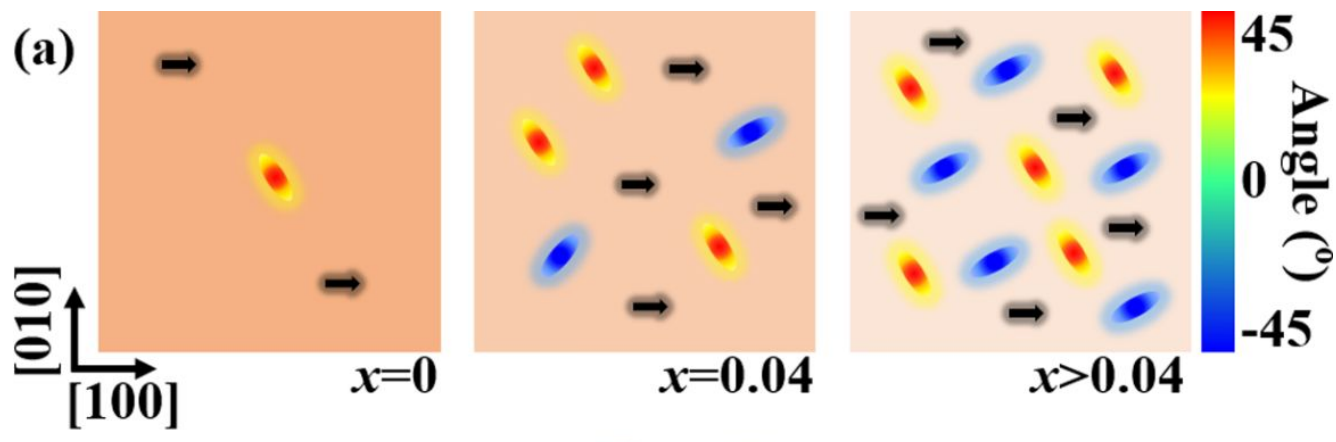

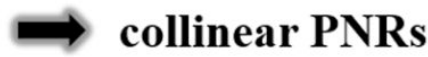

(b)

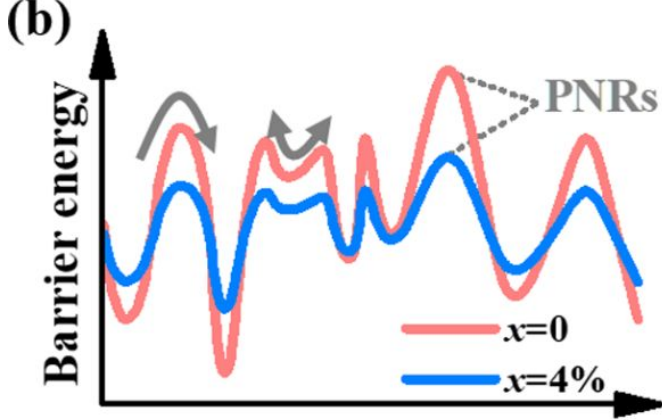

(d)
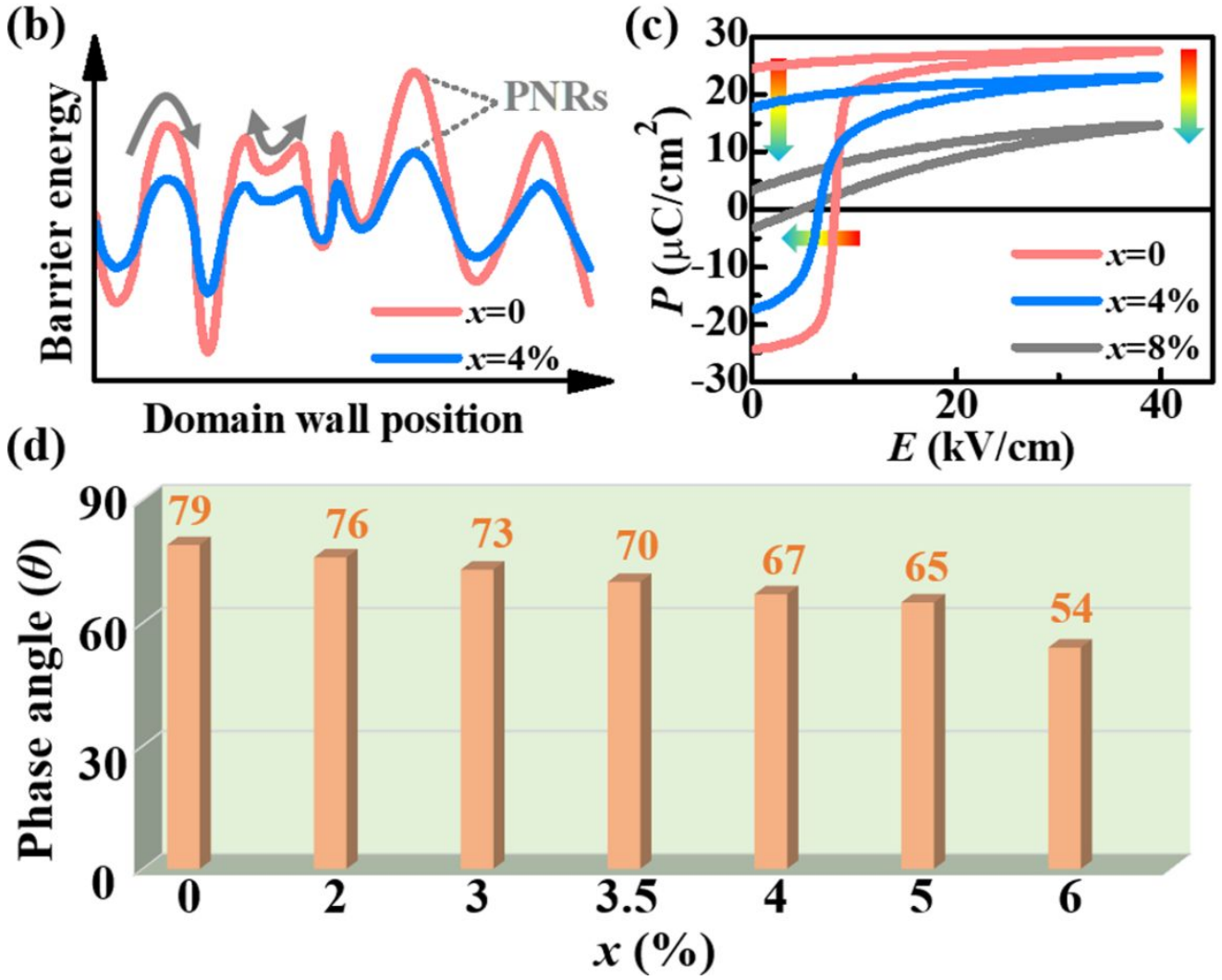

non-collinear PNRs

Figure 6. (a) Structural illustration of the distribution of ferroelectric domains and PNRs in poled KNNS-xBAZ-Fe 
ceramics; (b) schematic diagram of barrier energy for domain switching in KNNS- $x$ BAZ-Fe ( $x=0$ and 0.04$)$ ceramics; (c) $P$-E loops of KNNS- $x$ BAZ-Fe $(x=0,0.04$, and 0.08$)$ ceramics; (d) phase angle $(\theta)$ of poled KNNS- $x$ BAZ-Fe $(x=0-$ 0.06) ceramics. The fading color in (a) represents the decrease of ferroelectric domains in amount and size. Phase angle $(\theta)$ is extracted from Figure S17, ESI.

\section{Conclusion}

Here we exampled KNNS-xBAZ-Fe ceramics to reveal physical mechanisms of the enhanced piezoelectricity by PBE. We first obtained a high $d_{33}$ of $555 \mathrm{pC} / \mathrm{N}$ and the enhanced temperature stability of $S_{\text {uni }}$ at $x=0.04$. We then demonstrated that PBE-modified KNN-based ceramics (e.g., $x=0.04$ ) possess the R-O-T multi-phase coexistence with a structural softening. The structural softening is ascribed to the diffused R phase and the PNRs induced by aliovalent substitutions. TEM measurements show the abundant nano-domains with a scale of 10-80 nm, which are re-oriented by the electric field and then form the coexisting single-domain and multi-domain zones. The subsequent analysis shows that the increased $d_{33}$ hinges on the low polarization anisotropy, the flattened Gibbs free energy, and nano-domains. The first two enhance $\varepsilon_{\mathrm{r}}$ and the last one promotes the domain switching and domain wall motion. In particular, we revealed the "double-edged sword" role of PNRs. Although it facilitates the domain switching and domain wall motion, it cannot be re-orientated completely. Therefore, the increased $d_{33}$ is the balanced consequence of ferroelectric domains and PNRs at the R-O-T multi-phase coexistence. Finally, we also proposed a promising concept to further increase the $d_{33}$ of lead-free piezoceramics, that is, introducing a small number of PNRs to a ferroelectric matrix with very strong ferroelectricity. 


\section{Conflicts of interest}

The authors declare no competing interests.

\section{Acknowledgments}

The authors gratefully acknowledge the support of the National Natural Science Foundation of China (Nos. 51722208 and 51972215) and the Key Technologies Research and Development Program of Sichuan Province (No. 2018JY0007). Dr. X. Lv and Dr. J. Zhang contributed equally to this work.

\section{Reference}

(1) Rödel, J.; Webber, K. G.; Dittmer, R.; Jo, W.; Kimura, M.; Damjanovic, D. Transferring lead-free piezoelectric ceramics into application. J. Eur. Ceram. Soc. 2015, 35 (6), 1659-1681.

(2) Wu, J.; Xiao, D.; Zhu, J. Potassium-Sodium Niobate Lead-Free Piezoelectric Materials: Past, Present, and Future of Phase Boundaries. Chem. Rev. 2015, 115 (7), 2559-2595.

(3) Wang, X.; Wu, J.; Xiao, D.; Zhu, J.; Cheng, X.; Zheng, T.; Zhang, B.; Lou, X.; Wang, X. Giant piezoelectricity in potassium-sodium niobate lead-free ceramics. J. Am. Chem. Soc. 2014, 136 (7), 2905-2910.

(4) Xu, K.; Li, J.; Lv, X.; Wu, J.; Zhang, X.; Xiao, D.; Zhu, J. Superior Piezoelectric Properties in Potassium-Sodium Niobate Lead-Free Ceramics. Adv. Mater. 2016, 28 (38), 8519-8523.

(5) Li, P.; Zhai, J.; Shen, B.; Zhang, S.; Li, X.; Zhu, F.; Zhang, X. Ultrahigh Piezoelectric Properties in Textured (K,Na) $\mathrm{NbO}_{3}$-Based Lead-Free Ceramics. Adv. Mater. 2018, 30 (8), 1705171.

(6) Liu, Q.; Zhang, Y.; Gao, J.; Zhou, Z.; Wang, H.; Wang, K.; Zhang, X.; Li, L.; Li, J.-F. Highperformance lead-free piezoelectrics with local structural heterogeneity. Energy Environ. Sci. 2018, 
$11(12), 3531-3539$.

(7) Jiang, L.; Yang, Y.; Chen, R.; Lu, G.; Li, R.; Xing, J.; Shung, K. K.; Humayun, M. S.; Zhu, J.; Chen, Y. Ultrasound-Induced Wireless Energy Harvesting for Potential Retinal Electrical Stimulation Application. Adv. Funct. Mater. 2019, 29 (33), 1902522.

(8) Lv, X.; Zhu, J.; Xiao, D.; Zhang, X.-X.; Wu, J. Emerging new phase boundary in potassium sodiumniobate based ceramics. Chem. Soc. Rev. 2020, 49 (3), 671-707.

(9) Zheng, T.; Wu, J.; Cheng, X.; Wang, X.; Zhang, B.; Xiao, D.; Zhu, J.; Wang, X.; Lou, X. High strain in $\left(\mathrm{K}_{0.40} \mathrm{Na}_{0.60}\right)\left(\mathrm{Nb}_{0.955} \mathrm{Sb}_{0.045}\right) \mathrm{O}_{3}-\mathrm{Bi}_{0.50} \mathrm{Na}_{0.50} \mathrm{ZrO}_{3}$ lead-free ceramics with large piezoelectricity. J. Mater. Chem. C 2014, 2 (41), 8796-8803.

(10) Zhou, C.; Zhang, J.; Yao, W.; Wang, X.; Liu, D.; Sun, X. Piezoelectric performance, phase transitions, and domain structure of $0.96\left(\mathrm{~K}_{0.48} \mathrm{Na}_{0.52}\right)\left(\mathrm{Nb}_{0.96} \mathrm{Sb}_{0.04}\right) \mathrm{O}_{3}-0.04\left(\mathrm{Bi}_{0.50} \mathrm{Na}_{0.50}\right) \mathrm{ZrO}_{3}$ ceramics. J. Appl. Phys. 2018, 124 (16), 164101.

(11) Liu, D.; Zhang, X.; Su, W.; Wang, X.; Yao, W.; Zhou, C.; Zhang, J. Outstanding piezoelectric properties, phase transitions and domain configurations of $0.963\left(\mathrm{~K}_{0.48} \mathrm{Na}_{0.52}\right)\left(\mathrm{Nb}_{0.955} \mathrm{Sb}_{0.045}\right) \mathrm{O}_{3^{-}}$ 0.037( $\left.\mathrm{Bi}_{0.50} \mathrm{Na}_{0.50}\right) \mathrm{HfO}_{3}$ ceramics. J. Alloys. Compd. 2019, 779, 800-804.

(12) Zhang, J.; Sun, X.; Su, W.; Yao, W.; Zhou, C. Superior piezoelectricity and rhombohedralorthorhombic-tetragonal phase coexistence of $(1-x)(\mathrm{K}, \mathrm{Na})(\mathrm{Nb}, \mathrm{Sb}) \mathrm{O}_{3}-x(\mathrm{Bi}, \mathrm{Na}) \mathrm{HfO}_{3}$ ceramics. Scripta Mater. 2020, 176, 108-111.

(13) Li, P.; Chen, X.; Wang, F.; Shen, B.; Zhai, J.; Zhang, S.; Zhou, Z. Microscopic insight into electric fatigue resistance and thermally stable piezoelectric properties of $(\mathrm{K}, \mathrm{Na}) \mathrm{NbO}_{3}$-based ceramics. $A C S$ Appl. Mater. Interfaces 2018, 10 (34), 28772-28779.

(14) Li, P.; Huan, Y.; Yang, W.; Zhu, F.; Li, X.; Zhang, X.; Shen, B.; Zhai, J. High-performance 
potassium-sodium niobate lead-free piezoelectric ceramics based on polymorphic phase boundary and crystallographic texture. Acta Mater. 2019, 165, 486-495.

(15) Yang, W.; Li, P.; Wu, S.; Li, F.; Shen, B.; Zhai, J. A Study on the Relationship Between Grain Size and Electrical Properties in $(\mathrm{K}, \mathrm{Na}) \mathrm{NbO}_{3}$-Based Lead-Free Piezoelectric Ceramics. Adv. Electron. Mater. 2019, 5 (12), 1900570.

(16) Guo, H.; Zhou, C.; Ren, X.; Tan, X. Unique single-domain state in a polycrystalline ferroelectric ceramic. Phys. Rev. B 2014, 89 (10), 100104.

(17) Otonicar, M.; Park, J.; Logar, M.; Esteves, G.; Jones, J.; Jancar, B. External-field-induced crystal structure and domain texture in $(1-x) \mathrm{Na}_{0.5} \mathrm{Bi}_{0.5} \mathrm{TiO}_{3}-x \mathrm{~K}_{0.5} \mathrm{Bi}_{0.5} \mathrm{TiO}_{3}$ piezoceramics. Acta Mater. 2017, $127,319-331$

(18) Guo, H.; Voas, B. K.; Zhang, S.; Zhou, C.; Ren, X.; Beckman, S. P.; Tan, X. Polarization alignment, phase transition, and piezoelectricity development in polycrystalline $0.5 \mathrm{Ba}\left(\mathrm{Zr}_{0.2} \mathrm{Ti}_{0.8}\right) \mathrm{O}_{3^{-}}$ 0.5( $\left.\mathrm{Ba}_{0.7} \mathrm{Ca}_{0.3}\right) \mathrm{TiO}_{3}$. Phys. Rev. B 2014, 90 (1), 014103.

(19) Zakhozheva, M.; Schmitt, L.; Acosta, M.; Jo, W.; Rödel, J.; Kleebe, H.-J. In situ electric field induced domain evolution in $\mathrm{Ba}\left(\mathrm{Zr}_{0.2} \mathrm{Ti}_{0.8}\right) \mathrm{O}_{3}-0.3\left(\mathrm{Ba}_{0.7} \mathrm{Ca}_{0.3}\right) \mathrm{TiO}_{3}$ ferroelectrics. Appl. Phys. Lett. 2014, $105(11), 112904$.

(20) Zakhozheva, M.; Schmitt, L.; Acosta, M.; Guo, H.; Jo, W.; Schierholz, R.; Kleebe, H.-J.; Tan, X. Wide compositional range in situ electric field investigations on lead-free $\mathrm{Ba}\left(\mathrm{Zr}_{0.2} \mathrm{Ti}_{0.8}\right) \mathrm{O}_{3^{-}}$ $x\left(\mathrm{Ba}_{0.7} \mathrm{Ca}_{0.3}\right) \mathrm{TiO}_{3}$ piezoceramic. Phys. Rev. Appl. 2015, 3 (6), 064018.

(21) Fan, Z.; Zhou, C.; Ren, X.; Tan, X. Domain disruption and defect accumulation during unipolar electric fatigue in a BZT-BCT ceramic. Appl. Phys. Lett. 2017, 111 (25), 252902.

(22) Fan, Z.; Tan, X. In-situ TEM study of the aging micromechanisms in a $\mathrm{BaTiO}_{3}$-based lead-free 
piezoelectric ceramic. J. Eur. Ceram. Soc. 2018, 38 (10), 3472-3477.

(23) Li, F.; Zhang, S.; Yang, T.; Xu, Z.; Zhang, N.; Liu, G.; Wang, J.; Wang, J.; Cheng, Z.; Ye, Z. G.; Luo, J.; Shrout, T. R.; Chen, L. Q. The origin of ultrahigh piezoelectricity in relaxor-ferroelectric solid solution crystals. Nat. Commun. 2016, 7, 13807.

(24) Li, F.; Zhang, S.; Xu, Z.; Chen, L. Q. The Contributions of Polar Nanoregions to the Dielectric and Piezoelectric Responses in Domain-Engineered Relaxor-PbTiO ${ }_{3}$ Crystals. Adv. Funct. Mater. 2017, 27 (18), 1700310.

(25) Liu, L.; Knapp, M.; Schmitt, L. A.; Ehrenberg, H.; Fang, L.; Fuess, H.; Hoelzel, M.; Hinterstein, M. Structure and dielectric dispersion in cubic-like $0.5 \mathrm{~K}_{0.5} \mathrm{Na}_{0.5} \mathrm{NbO}_{3}-0.5 \mathrm{Na}_{1 / 2} \mathrm{Bi}_{1 / 2} \mathrm{TiO}_{3}$ ceramic. $E P L$ (Europhys. Lett.) 2016, 114 (4), 47011.

(26) Sun, X.; Zhang, J.; Lv, X.; Liu, Y.; Li, F.; Wu, J. Understanding Piezoelectricity of HighPerformance Potassium Sodium Niobate Ceramics from Diffused Multi-phase Coexistence and Domain Feature. J. Mater. Chem. A 2019, 7 (28), 16803-16811.

(27) Tao, H.; Wu, H.; Liu, Y.; Zhang, Y.; Wu, J.; Li, F.; Lyu, X.; Zhao, C.; Xiao, D.; Zhu, J. Ultrahigh Performance in Lead-free Piezoceramics Utilizing a Relaxor Slush Polar State with Multiphase Coexistence. J. Am. Chem. Soc. 2019, 141 (35), 13987-13994.

(28) Liu, W.; Ren, X. Large piezoelectric effect in Pb-free ceramics. Phys. Rev. Lett. 2009, 103 (25), 257602.

(29) Zhao, C.; Wu, H.; Li, F.; Cai, Y.; Zhang, Y.; Song, D.; Wu, J.; Lyu, X.; Yin, J.; Xiao, D. Practical High Piezoelectricity in Barium Titanate Ceramics Utilizing Multiphase Convergence with Broad Structural Flexibility. J. Am. Chem. Soc. 2018, 140 (45), 15252-15260.

(30) Wu, B.; Wu, H.; Wu, J.; Xiao, D.; Zhu, J.; Pennycook, S. J. Giant piezoelectricity and high Curie 
temperature in nanostructured alkali niobate lead-free piezoceramics through phase coexistence. $J$. Am. Chem. Soc. 2016, 138 (47), 15459-15464.

(31) Lee, M. H.; Kim, D. J.; Park, J. S.; Kim, S. W.; Song, T. K.; Kim, M. H.; Kim, W. J.; Do, D.; Jeong, I. K. High-performance lead-free piezoceramics with high curie temperatures. Adv. Mater. 2015, 27 (43), 6976-6982.

(32) Wu, J. Advances in Lead-free Piezoelectric Materials, Springer: 2018.

(33) Yang, Y.; Ji, Y.; Fang, M.; Zhou, Z.; Zhang, L.; Ren, X. Morphotropic Relaxor Boundary in a Relaxor System Showing Enhancement of Electrostrain and Dielectric Permittivity. Phys. Rev. Lett. 2019, 123 (13), 137601.

(34) Zhang, S.-T.; Kounga, A. B.; Aulbach, E.; Jo, W.; Granzow, T.; Ehrenberg, H.; Rödel, J. Leadfree piezoceramics with giant strain in the system $\mathrm{Bi}_{0.5} \mathrm{Na}_{0.5} \mathrm{TiO}_{3}-\mathrm{BaTiO}_{3}-\mathrm{K}_{0.5} \mathrm{Na}_{0.5} \mathrm{NbO}_{3}$. II. Temperature dependent properties. J. Appl. Phys. 2008, 103 (3), 034108-1.

(35) Zhao, C.; Wu, B.; Thong, H.-C.; Wu, J. Improved temperature stability and high piezoelectricity in lead-free barium titanate-based ceramics. J. Eur. Ceram. Soc. 2018, 38 (16), 5411-5419.

(36) Saito, Y.; Takao, H.; Tani, T.; Nonoyama, T.; Takatori, K.; Homma, T.; Nagaya, T.; Nakamura, M. Lead-free piezoceramics. Nature 2004, 432 (7013), 84-87.

(37) Wang, K.; Yao, F. Z.; Jo, W.; Gobeljic, D.; Shvartsman, V. V.; Lupascu, D. C.; Li, J. F.; Rödel, J. Temperature-Insensitive (K,Na) $\mathrm{NbO}_{3}$-Based Lead-Free Piezoactuator Ceramics. Adv. Funct. Mater. 2013, 23 (33), 4079-4086.

(38) Zhang, M.-H.; Wang, K.; Zhou, J.-S.; Zhou, J.-J.; Chu, X.; Lv, X.; Wu, J.; Li, J.-F. Thermally stable piezoelectric properties of $(\mathrm{K}, \mathrm{Na}) \mathrm{NbO}_{3}$-based lead-free perovskite with rhombohedraltetragonal coexisting phase. Acta Mater. 2017, 122, 344-351. 
(39) Brajesh, K.; Tanwar, K.; Abebe, M.; Ranjan, R. Relaxor ferroelectricity and electric-field-driven structural transformation in the giant lead-free piezoelectric $(\mathrm{Ba}, \mathrm{Ca})(\mathrm{Ti}, \mathrm{Zr}) \mathrm{O}_{3}$. Phys. Rev. B 2015, 92 (22), 224112.

(40) López-Juárez, R.; Novelo-Peralta, O.; González-García, F.; Rubio-Marcos, F. Ferroelectric domain structure of lead-free potassium-sodium niobate ceramics. J. Eur. Ceram. Soc. 2011, 31 (9), 1861-1864.

(41) Ding, Y.; Zheng, T.; Zhao, C.; Wu, J. Structure and domain wall dynamics in lead-free KNNbased ceramics. J. Appl. Phys. 2019, 126 (12), 124101.

(42) Hu, C.; Meng, X.; Zhang, M.-H.; Tian, H.; Daniels, J. E.; Tan, P.; Huang, F.; Li, L.; Wang, K.; Li, J.-F.; Lu, Q.; Cao, W.; Zhou, Z. Ultra-large electric field-induced strain in potassium sodium niobate crystals. Sci. Adv. 2020, 6 (13), eaay5979.

(43) Wang, Y.; Luo, C.; Wang, S.; Chen, C.; Yuan, G.; Luo, H.; Viehland, D. Large Piezoelectricity in Ternary Lead-Free Single Crystals. Adv. Electron. Mater. 2020, 6 (1), 1900949.

(44) Schmitt, L. A.; Schönau, K. A.; Theissmann, R.; Fuess, H.; Kungl, H.; Hoffmann, M. J. Composition dependence of the domain configuration and size in $\mathrm{Pb}\left(\mathrm{Zr}_{1-x} \mathrm{Ti}_{x}\right) \mathrm{O}_{3}$ ceramics. J. Appl. Phys. 2007, 101 (7), 074107.

(45) Li, J.; Fei, L.; Zhang, S. Decoding the Fingerprint of Ferroelectric Loops: Comprehension of the Material Properties and Structures. J. Am. Ceram. Soc. 2014, 97 (1), 1-27.

(46) Uchino, K.; Nomura, S.; Cross, L. E.; Newnham, R. E.; Jang, S. J. Electrostrictive effect in perovskites and its transducer applications. J. Mater. Sci. 1981, 16 (3), 569-578.

(47) Damjanovic, D. Contributions to the piezoelectric effect in ferroelectric single crystals and ceramics. J. Am. Ceram. Soc. 2005, 88 (10), 2663-2676. 
(48) Fu, H.; Cohen, R. E. Polarization rotation mechanism for ultrahigh electromechanical response in single-crystal piezoelectrics. Nature 2000, 403 (6767), 281.

(49) Zhang, S.; Li, F. High performance ferroelectric relaxor- $\mathrm{PbTiO}_{3}$ single crystals: Status and perspective. J. Appl. Phys. 2012, 111 (3), 2.

(50) Gao, J.; Hao, Y.; Ren, S.; Kimoto, T.; Fang, M.; Li, H.; Wang, Y.; Zhong, L.; Li, S.; Ren, X. Large piezoelectricity in $\mathrm{Pb}$-free $0.96\left(\mathrm{~K}_{0.5} \mathrm{Na}_{0.5}\right)_{0.95} \mathrm{Li}_{0.05} \mathrm{Nb}_{0.93} \mathrm{Sb}_{0.07} \mathrm{O}_{3}-0.04 \mathrm{BaZrO}_{3}$ ceramic: $\mathrm{A}$ perspective from microstructure. J. Appl. Phys. 2015, 117 (8), 084106.

(51) Li, F.; Cabral, M. J.; Xu, B.; Cheng, Z.; Dickey, E. C.; LeBeau, J. M.; Wang, J.; Luo, J.; Taylor, S.; Hackenberger, W. Giant piezoelectricity of Sm-doped $\mathrm{Pb}\left(\mathrm{Mg}_{1 / 3} \mathrm{Nb}_{2 / 3}\right) \mathrm{O}_{3}-\mathrm{PbTiO}_{3}$ single crystals. Science 2019, 364 (6437), 264-268. 